\title{
Rendimento escolar na matemática: Efeito diferencial da ansiedade e das
}

\author{
crenças de autoeficácia
}

\section{School achievement in mathematics: Differential effect of anxiety and self- efficacy beliefs}

\author{
José Tomás da Silva, Maria Paula Paixão, Teresa Sousa Machado, José Pacheco Miguel \\ Universidade de Coimbra
}

\begin{abstract}
Resumo
A matemática tem sido perspetivada como um "filtro crítico" para o acesso a carreiras científicas e tecnológicas. Muitas variáveis têm sido identificadas como preditores do rendimento na matemática, designadamente a ansiedade $\mathrm{e}$ autoeficácia matemática. Neste estudo, com 112 alunos do ensino secundário examinamos o papel destas variáveis depois de controlarmos o rendimento prévio e o sexo dos estudantes, no desempenho escolar da matemática numa prova intermédia sumativa. Uma regressão linear mostrou que a ansiedade é um preditor mais eficiente do que a medida de autoeficácia do rendimento a matemática. As implicações psicopedagógicas dos resultados são discutidas.
\end{abstract}

Palavras-chave: autoeficácia, ansiedade, realização a matemática

\begin{abstract}
Mathematics has been described as a "critical filter" for access to scientific and technological careers. Many variables have been identified as predictors of mathematical achievement, namely, anxiety and mathematical self-efficacy. In this study, with 112 secondary school students, we examined the role of these variables, after controlling the students' previous performance and sex, in the school performance of mathematics in a summative intermediate test. A linear regression showed that anxiety is a more efficient predictor than the measure of self-efficacy of mathematical performance. The psychoeducational implications of the results are discussed.
\end{abstract}

Keywords: self-efficacy, anxiety, mathematics achievement

A matemática tem sido perspetivada como um "filtro crítico" (Sells, 1980) para o acesso a carreiras científicas e tecnológicas, domínios profissionais tradicionalmente masculinos e, simultaneamente, associados a um maior prestígio e remunerações mais elevadas (Brown \& Lent, 2016). As profissões incluídas no grupo das disciplinas ditas STEM (acrónimo anglo-saxónico referente às disciplinas Científicas, Tecnológicas, Engenharias e Matemáticas) encontram-se, internacionalmente, no topo da lista das profissões com perspetivas de desenvolvimento mais brilhantes para os próximos anos (vide $\mathrm{O} *$ Net OnLine em www.onetonline.org/find/ bright? $\mathrm{b}=1 \& \mathrm{~g}=\mathrm{Go})$. Obter êxito a matemática é um dos mais importantes requisitos para que os indivíduos possam prosseguir uma formação superior na área das disciplinas STEM, e, consequentemente para obterem uma colocação profissional nesses domínios de atividade. Como foi sublinhado por Pajares e Graham (1999) a matemática continua a ocupar um lugar proeminente entre as medidas de avaliação do desempenho académico usadas nos processos de colocação dos candidatos em programas de estudos altamente competitivos e na admissão a vários cursos do ensino superior. Infelizmente sucessivos relatórios, quer internos (produzidos por iniciativa de um único país), quer externos (i.e., aqueles promovidos e realizados por organizações supranacionais, como por exemplo, a OCDE) continuam a mostrar um desfasamento entre a oferta e a procura no mercado de competências científicas e tecnológicas. Todavia, ninguém duvida que o domínio de competências matemáticas avançadas é crucial para o desempenho competitivo da força de trabalho de qualquer país no contexto atual de concorrência industrial e comercial cada vez mais globalizada. Na literatura sobre este tema frequentemente transparece a ideia de que, por muitas e variadas razões, durante o transcurso escolar o sistema vai perdendo muitos dos alunos potenciais para os domínios científicos e tecnológicos. Nos EUA esta "perda na canalização" (pipeline leak) educativa está suficientemente documentada para grupos específicos da população (e.g., indivíduos do sexo feminino e minorias raciais e étnicas). Por causa disso, este problema é atualmente perspetivado pelas autoridades e instituições políticas nos EUA não apenas como um problema geral de oferta-procura, mas também como uma questão de justiça social, na medida em que as mulheres e membros de minorias raciais/étnicas continuam a estar sub-representadas em áreas educativas e profissionais de tipo STEM (Brown \& Lent, 2016). Por outro lado, sabemos que muitos estudantes com bons conhecimentos matemáticos durante o ensino secundário mostram, paradoxalmente, uma realização académica em exames e provas estandardizadas inferiores ao previsto com base nas suas aptidões e capacidades cognitivas. Algumas variáveis 
têm sido historicamente identificadas como tendo um papel crucial neste desfasamento entre capacidade e performance, particularmente, a ansiedade e autoeficácia matemática tem sido frequentemente mencionadas na literatura científica a este respeito (Bandura, 1997; Betz \& Hackett, 1983; Caprara \& Cervone, 2000; Fouad \& Smith, 1996; Hackett, 1985; Lent, Lopez, \& Bieschke, 1993; Pajares \& Miller, 1994; Zeidner, 1998).

Historicamente, na Psicologia, o estudo do papel da ansiedade (especialmente da ansiedade aos exames) precede os trabalhos que usam preferencialmente o construto de autoeficácia (e.g., Zeidner, 1998; 2004; Zeidner \& Mathews, 2005). Embora, como referem Zeidner e colaboradores (2004, 2005), stress e ansiedade sejam experiências humanas universais, nas sociedades modernas os estímulos ambientais que desencadeiam estas emoções mudaram radicalmente. De facto, os exames e as situações de avaliação, em geral, emergiram como uma potente classe de estímulos evocadores dessas respostas, tendo em conta que muitas decisões importantes relativas à condição dos indivíduos na escola, na universidade, no emprego, se baseiam em exames, testes e muitos outros procedimentos de avaliação (Zeidner, 2004). A ansiedade matemática inclui-se entre as diversas modalidades específicas de ansiedade avaliativa (e.g., ansiedade aos exames, ansiedade informática e aos computadores, ansiedade social, ansiedade no desporto) que têm atraído uma especial atenção dos investigadores. A ansiedade matemática foi definida como os "sentimentos de tensão, desamparo, desorganização mental e sintomas corporais associados que são evocados nas situações de resolução de problemas matemáticos” (Zeidner \& Matthews, 2005, p. 141). Ao longo dos anos foram propostos vários modelos e conceitos teóricos ligando a ansiedade avaliativa e a competência/performance em diferentes domínios da vida. No caso específico da ansiedade matemática a evidência acerca da intensidade da sua relação com o rendimento/realização pode extrair-se de um dos estudos de meta-análise que se ocupou deste tópico (e.g., Schwarzer, Seipp, \& Schwarzer, 1989). Dos 47 efeitos (coeficientes de correlação) observados, Schwarzer et al. (1989) obtiveram um $r=-.23$ como a melhor estimação populacional da ligação ansiedade matemática-performance. Estudos realizados em laboratório, bem como outros efetuados em situações de avaliação reais, mostram que a ansiedade interfere na realização competente de muitas tarefas. As centenas de estudos publicados revelaram vários défices de processamento que estão relacionados com a ansiedade, nomeadamente deficiências gerais da atenção e da memória de trabalho que se conjugam com mudanças performativas mais impercetíveis como as implicadas na organização eficiente da informação semântica (Zeidner \& Matthews, 2005). Interpretações mais recentes dos resultados das investigações sugerem haver vantagem em perspetivar a natureza da associação ansiedadeperformance como inerentemente recíproca. Elevados níveis de ansiedade, segundo Zeidner (2004; Zeidner \& Matthews, 2005), começam por absorver parte dos recursos necessários para os processos de atenção, memória de curto prazo, resolução de problemas ou outros processos cognitivos requeridos para a execução bem-sucedida duma tarefa avaliativa concreta (e.g., resolver um teste de problemas de matemática; tocar a sonata para piano n. ${ }^{\circ} 9$ de Beethoven num concerto). Admite-se ainda que a ansiedade avaliativa produz igualmente alguns padrões motivacionais aversivos, de enfrentamento (coping) e estratégias associadas com a tarefa que interferem com a aprendizagem e a realização. Em consequência o sentimento de competência e de autoeficácia sofre ainda mais, conduzindo a mais ansiedade ao longo do tempo e dando origem a um ciclo vicioso de crescente ansiedade e de degradação da competência (Zeidner, 2004). Esta perspetiva dinâmica (admitindo a atuação de mecanismos de autorregulação ao longo do tempo), considerada atualmente pelos principais teóricos da ansiedade avaliativa como essencial para se compreender a natureza recíproca da relação ansiedade-performance foi desde o início privilegiada pelos proponentes da abordagem social cognitiva do comportamento (e.g., Bandura, 1986). A teoria sociocognitiva de Bandura (1986) e, especialmente, a componente relativa à autoeficácia (Bandura, 1997), tem-se revelado bastante heurística na elucidação dos processos de autorregulação e de motivação em contextos académicos e dos respetivos contributos para o rendimento nas diferentes áreas escolares, designadamente na matemática (Pajares, 1996). As crenças de autoeficácia, isto é, os "julgamentos da pessoa sobre a sua capacidade para organizar e executar os cursos de ação necessários para atingir um tipo designado de desempenhos" (Bandura, 1986, p. 391), como mostram numerosos estudos, contribuem para aumentar as realizações e o bem-estar dos indivíduos de inúmeras maneiras. Desde logo, como sugere Pajares (2005), as crenças de autoeficácia influenciam as escolhas que as pessoas fazem e os cursos de ação que adotam. As crenças de autoeficácia, por outro lado, ajudam a determinar o esforço que as pessoas irão empregar numa atividade, por quanto tempo irão perseverar quando confrontadas com obstáculos e o grau de resiliência que demostrarão em situações de adversidade (Pajares, 2005). Finalmente, as crenças de autoeficácia também influenciam os padrões de pensamento e as reações emocionais dos indivíduos. Os efeitos positivos das crenças de autoeficácia na ação humana estão amplamente documentados nos numerosos estudos de meta-análise e nas revisões de literatura até agora realizadas (e.g., Bandura, 1997; Richardson, Abraham \& Bond, 2012; Robbins, Lauver, Le, Davis, Langley \& Carlstrom, 2004).

Há uma literatura abundante mostrando que as pessoas com elevada ansiedade avaliativa têm baixas expetativas de controlo pessoal especialmente a nível da autoeficácia e expetativas de resultado e que estas variáveis estão mais fortemente relacionadas com o sucesso performativo do que outras variáveis mais generalistas do self, como o autoconceito e a autoestima (Caprara \& Cervone, 2000; Zimmerman, 2000). Tanto a autoeficácia como as expetativas de resultado têm sido designadas como variáveis percursoras da ansiedade matemática (e.g., Hackett, 1985; Schwarzer \& Jerusalem, 1992; Schwarzer, Mueller, \& Greenglass, 1999). Já Smith, 
Arnkoff e Wright (1990) confirmaram que a autoeficácia contribuía para a predição da ansiedade avaliativa, acima e além da contribuição da interferência cognitiva e das deficiências detetadas nas habilidades de estudo. Da mesma forma Betz e Hackett (1983) mostraram que a perceção da eficácia associada à matemática predizia a ansiedade matemática, mesmo depois de controlado o efeito do rendimento prévio a matemática. Ambos os estudos referidos por último foram realizados com estudantes universitários e importa verificar se os seus resultados se podem generalizar a amostras de alunos mais novos. Desta forma, um dos objetivos deste estudo é examinar o papel diferencial da autoeficácia matemática relativamente ao rendimento escolar anterior na predição da ansiedade matemática. Alguma investigação no domínio da psicologia vocacional (e.g., Brown \& Lent, 2016; Hackett \& Betz, 1989; Fouad \& Smith, 1996), por outro lado, tem procurado identificar diferenças sexuais nas ligações entre os construtos incluídos neste estudo. Assim, na linha dos trabalhos de Farmer, Wardrop, Anderson e Risinger (1995) e Hackett (1985) ir-se-á testar, primeiramente, qual a ligação da variável sexo com as outras variáveis incluídas no modelo preditivo.

\section{Método}

\section{Participantes}

Os dados usados neste estudo provêm de uma amostra não-probabilística de 112 estudantes que, no momento em que foram contactados, frequentavam o $10^{\circ}$ ano de escolaridade (ensino secundário) na região do Algarve. Do total da amostra, $n=53$ (ou seja, 47\%) são do sexo masculino e os restantes $(n=60,63 \%)$ são do sexo feminino. A média de idade dos participantes, para o total da amostra, é de 15.2 anos $(D P=0.68)$.

\section{Procedimento}

A administração do inquérito foi efetuada pelo primeiro autor, em tempos letivos concedidos pelos docentes das disciplinas contactados, que permanecerem na sala de aula enquanto decorreu o preenchimento dos questionários, embora não interferissem na administração do mesmo. A realização do estudo foi autorizada pelo Conselho Executivo e integrou o plano de atividades do Serviço de Psicologia e Orientação (SPO) do estabelecimento de ensino onde decorreu o estudo. Para a investigação apenas foram contactadas as turmas do $10^{\circ}$ ano de escolaridade que, no plano de estudos, incluíam a disciplina de Matemática A. Os encarregados de educação autorizaram a participação dos respetivos educandos no estudo e os participantes foram completamente esclarecidos acerca dos objetivos do estudo e do caráter confidencial das suas respostas. Todos os alunos contactados assentiram em participar. $\mathrm{O}$ tempo necessário para a recolha dos dados foi de aproximadamente trinta minutos.

\section{Instrumentos e medidas}

Um breve questionário de tipo papel-e-lápis foi desenvolvido para recolher a informação necessária para a mensuração das variáveis-alvo deste estudo. Os alunos começaram por fornecer alguns dados sociodemográficos e de seguida responderam a dois instrumentos de autorrelato. Foi-lhes ainda pedido que reportassem a nota de matemática que obtiveram no final do $9 .^{\circ}$ ano de escolaridade (sistema de classificação na escala de $1=$ fraco a $5=$ muito bom ou excelente). Posteriormente, no final do $1 .^{\circ}$ período, foi obtida a nota escolar a matemática de cada aluno (escala de 0 (zero) a 20 valores).

Mathematics Anxiety Scale (MAS). A versão traduzida para o Português Europeu da MAS (Betz, 1978) inclui 10 itens (e.g., "Quase nunca fico ansiosa/o quando estou a fazer testes de matemática") que são respondidos numa escala de tipo Likert com 5 pontos (1 = Discordo fortemente $5=$ Concordo fortemente). A escala visa medir sentimentos de ansiedade, medo, nervosismo e sintomas corporais relacionados com a realização a matemática (Betz, 1978). As respostas foram pontuadas de modo a que scores mais elevados correspondessem a maiores níveis de ansiedade matemática. A estimativa de consistência interna (estimada pelo método Split-half) reportada por Betz (1978) foi .92. Evidência acerca da validade dos resultados da MAS pode deduzir-se do padrão de associações encontrado com outras variáveis psicológicas (e.g., ansiedade traço). A estimativa da consistência interna obtida para os dados desta amostra foi $\alpha=.87$.

Autoeficácia Temas de Matemática A. Trata-se de um instrumento, desenvolvido pelo primeiro autor deste estudo, incluindo 14 itens (e.g., "Indique o seu grau de confiança para resolver: Problemas relacionados com a geometria no plano e no espaço") respondidos numa escala de tipo Likert com 7 pontos $(1=N a d a$, ou pouco confiante 7 = Muito, ou totalmente confiante). Esta escala foi construída para medir o grau de confiança do respondente sobre a sua capacidade para resolver com sucesso os problemas de matemática que lhe serão propostos, dentro de cada tema de Matemática A, ao longo do ano letivo. As respostas foram pontuadas de forma que scores mais altos correspondam a maiores níveis de autoeficácia matemática. O coeficiente alfa de Cronbach para as respostas desta amostra foi de .92. Evidência acerca da validade de construto, designadamente sobre a dimensionalidade das respostas, foi obtida através da análise fatorial (Silva, 2011).

\section{Resultados}

Começámos por realizar o estudo do efeito diferencial do sexo no conjunto das variáveis de resultado deste estudo, uma vez que a resposta a esta primeira questão condicionaria a natureza do modelo preditivo final. Uma MANOVA foi executada nas variáveis quatro variáveis quantitativas: nota a matemática (9. ${ }^{\circ}$ ano), nota a matemática (10. ${ }^{\circ}$ ano), score de ansiedade matemática (MAS) e score de autoeficácia para aprendizagem dos temas de matemática. $\mathrm{O}$ efeito multivariado para o sexo não revelou ser estatisticamente significativo: $(\Lambda=.94)$, $F(4,107)=1.80, p=14$. Este resultado é igualmente consistente com as correlações de Pearson (ver Tabela 1) do sexo $(1=$ masculino, $2=$ feminino $)$ com as restantes 
variáveis do estudo. Donde, seguidamente decidiu-se prosseguir as análises usando a amostra global (i.e., combinando os dois sexos).

Tabela 1.

Médias, desvios-padrão e correlações de Pearson ${ }^{a}$

\begin{tabular}{|c|c|c|c|c|c|c|}
\hline & $M$ & $D P$ & 1 & 2 & 3 & 4 \\
\hline 1. Nota $10^{\circ}$ & 11.2 & 3.8 & & & & \\
\hline 2. Autoeficácia & 61.2 & 13.7 & .48 & & & \\
\hline 3. Ansiedade & 29.3 & 7.7 & -.47 & -.62 & & \\
\hline 4. Nota $9^{\circ}$ & 3.7 & 0.8 & .54 & .53 & -.35 & \\
\hline 5. Sexo & - & - & .14 & -.09 & .10 & .05 \\
\hline
\end{tabular}

O padrão das correlações entre as variáveis é teoricamente consistente. As duas variáveis do rendimento escolar a matemática estão positiva e fortemente relacionadas. A classificação final a matemática no $9 .^{\circ}$ ano está positiva e fortemente associada com a autoeficácia matemática. Já a sua associação com ansiedade matemática é forte e negativa. A ansiedade e a autoeficácia matemáticas, por sua vez, mostram estar fortemente e inversamente correlacionadas (a percentagem de variância comum é aproximadamente $38 \%$ ). Finalmente, estas duas variáveis apresentam correlações de ordem zero bastante parecidas (embora, distintas quanto à direccionalidade) com a classificação dos alunos a matemática no fim do $1^{\circ}$ período do $100^{\circ}$ ano.

Tendo-se verificado que as variáveis métricas não mostravam desvios significativos da normalidade a matriz de variâncias-covariâncias das variáveis manifestas foi usada para estimar, através do método de máxima verosimilhança $(M L)$, implementado no software IBM AMOS, os parâmetros estruturais que constam na Figura 1. O modelo apresenta graus de liberdade nulos e por isso não pode ser testado quanto à significância estatística.

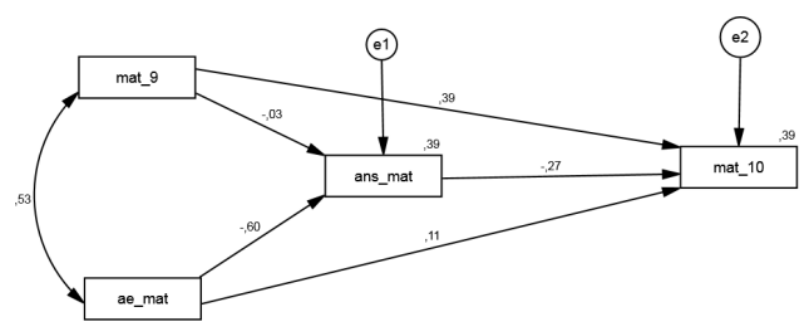

Figura 1. Modelo estrutural completo dos conhecimentos matemáticos, autoeficácia e ansiedade matemática no rendimento a matemática.

Legenda: mat_9 $=$ nota $9^{\circ}$ ano; ae_mat $=$ autoeficácia matemática; ans_mat $=$ ansiedade matemática; mat_ $10=\operatorname{nota} 10^{\circ}$ ano.

O modelo completo explica $39 \%$ da variância total dos resultados a matemática $\left(10^{\circ}\right.$ ano $)$ e $39 \%$ da variância na ansiedade matemática é explicada conjuntamente pela nota prévia a matemática $\left(9^{\circ}\right.$ ano, $\left.\beta=-.03, p>.05\right)$ e pela autoeficácia matemática $(\beta=-.60, p<.001)$. A nota a matemática no $9^{\circ}$ ano $(\beta=.39, p<.001)$ e a ansiedade matemática $(\beta=-.27, p<.01)$ são ambos preditores estatisticamente significativos do rendimento a matemática no $10^{\circ}$ ano, enquanto que a autoeficácia matemática $(\beta=.11, p>.05)$ não apresenta um efeito direto, estatisticamente significativo, na realização matemática no $10^{\circ}$ ano de escolaridade.

\section{Discussão}

O nível de realização escolar dos alunos é influenciado por inúmeros fatores de distinta natureza (e.g., socioeconómicos, cognitivos, afetivos). Compreender porque alguns alunos têm êxito nas tarefas escolares enquanto outros falham nas mesmas é um exercício que continua a captar a atenção e o esforço de académicos e investigadores que reconhecem a importância crucial de uma educação sólida para futuros empreendimentos em muitas áreas da vida. Neste estudo cingimo-nos ao exame do efeito conjunto de um tipo de disposição de personalidade contextualizada (ansiedade matemática) e de um fator cognitivo-motivacional (autoeficácia matemática) no desempenho a matemática, no começo dos estudos secundários. O domínio da matemática foi escolhido porque a matemática continua a ser uma disciplina charneira na admissão a vários cursos do ensino superior (Pajares \& Graham, 1999), nomeadamente dos associados às áreas STEM, ademais sendo os que apresentam perspetivas de empregabilidade mais brilhantes. Mencionámos o fenómeno de "vazamento" na conduta (pipeline) que conduz a estas áreas e, em especial, frisámos que isso afeta sobretudo as mulheres e membros de etnias minoritárias e marginalizadas. Argumentámos ainda que muito talento científico e matemático é desperdiçado neste processo e o nosso estudo procurou examinar dois fatores que regulam esse resultado. Os resultados que encontrámos, embora precisem de ser replicados em outras investigações independentes, mostram que, tal como prevíamos, e, como a investigação tem documentado (Bandura, 1997; Hackett, 1995) a autoeficácia para aprender conceitos matemáticos tem um efeito forte na ansiedade matemática que, por sua vez, afeta negativamente a realização a matemática. $O$ efeito da autoeficácia é totalmente mediado pela ansiedade matemática, enquanto o capital de conhecimento adquirido previamente (aqui inferido com base na classificação a matemática no final do terceiro ciclo) continua a ter um efeito direto forte no rendimento escolar futuro, mesmo quando o efeito das expetativas e das disposições analisadas são estatisticamente controladas. Estes dados têm várias implicações para a intervenção psicopedagógica e vocacional. Considerando que competências matemáticas e científicas são importantes para o sucesso em muitos campos profissionais, muitos esforços têm sido dirigidos para identificar as variáveis chave que aumentam o interesse dos alunos pela matemática e as áreas científicas com a esperança de que esse aumento do interesse conduza subsequentemente a um aumento da performance nestas áreas. Investigações e recensões anteriores (e.g., Luzzo et al., 1990; Pajares, 2005) mostram que é possível mudar as crenças de autoeficácia atuando sobre as fontes que as sustentam (especialmente as realizações de desempenho 
e experiências vicariantes) e que estas mudanças têm os efeitos desejados nos comportamentos-alvo relevantes: interesse, decisões e performance. Esta investigação mostra ainda que, embora as intervenções dirigidas para a gestão e o controlo da ansiedade sejam importantes esse não é o único mecanismo que precisa de ser modificado. Alterar as perceções/crenças de eficácia pessoal é um caminho valioso para intervenção neste campo considerando que estas, como sublinhou Bandura, tocam praticamente todos os aspetos das vidas das pessoas.

\section{Referências}

Bandura, A. (1986). Social foundations of thought and action: A social cognitive theory. Englewood Cliffs, NJ: Prentice Hall.

Bandura, A. (1997). Self-efficacy: The exercise of control. New York: Freeman.

Betz, N. E., \& Hackett, G. (1983). The relationship of mathematics self-efficacy expectations to the selection of science-based college majors. Journal of Vocational Behavior, 23, 329-345.

Brown, S. D. \& Lent, R. W. (2016). Vocational psychology: Agency, equity, and well-being. Annual Review of Psychology, 67, 541-565. doi: 10.1146/annurev-psych-122414-033237

Caprara, G. V., \& Cervone, D. (2000). Personality: determinants, dynamics, and potentials. Cambridge, UK: Cambridge University Press.

Farmer, H. S., Wardrop, J. L., Anderson, M. Z., \& Risinger, R. (1995). Women's career choices: Focus on science, math, and technology careers. Journal of Counseling Psychology, 42, 155-170.

Hackett, G. (1985). The role of mathematics self-efficacy in the choice of math-related majors of college women and men: A path analysis. Journal of Counseling Psychology, 32, 47-56.

Hackett, G., \& Betz, N. E. (1989). An exploration of the mathematics self-efficacy/mathematics performance correspondence. Journal of Research in Mathematics Education, 20, 261-273.

Fouad, N. A. \& Smith, P. L. (1996). A test of a social cognitive model for middle school students: Math and science. Journal of Counseling Psychology, 43, 338346.

Pajares, F. (2005). Self-efficacy during childhood and adolescence: Implications for teachers and parents. In F. Pajares \& T. Urdan (Eds.), Self-efficacy beliefs of adolescents (pp. 339-367). Information Age Publishing

Pajares, F. \& Graham, L. (1999). Self-efficacy, motivation constructs, and mathematics performance of entering middle school students. Contemporary Educational Psychology, 24, 124-139.

Pajares, F., \& Miller, M. D. (1994). The role of selfefficacy and self-concept beliefs in mathematical problem-solving: A path analysis. Journal of Educational Psychology, 86, 193-203.

Lent, R. W., Lopez, F. G., \& Bieschke, K.J. (1993). Predicting mathematics-related choice and success behaviors: Test of an expanded social cognitive model. Journal of Vocational Behavior, 42, 223-236.
Luzzo, D. A., Hasper, P., Alpert, K. A., Bibby, M., \& Martinelli, E. (1990). Effects of self-efficacyenhancing interventions on the math/science selfefficacy and career interests, goals and actions of career undecided college students. Journal of Counseling Psychology, 46, 233-243.

Richardson, Abraham \& Bond, (2012). Psychological correlates of university students' academic performance: A systematic review and meta-analysis. Psychological Bulletin, 138, 353-387. doi: 10.1037/a0026838

Robbins, S. B., Lauver, K., Le, H., Davis, D., Langley, R., \& Carlstrom, A. (2004). Do psychosocial and study skill factors predict college outcomes? A metaanalysis. Psychological Bulletin, 130, 261-288. doi: 10.1037/0033-2909.130.2.261

Schwarzer, R., \& Jerusalem, M. (1992). Advances in anxiety theory: A cognitive process approach. In K. A. Hagtvet \& T. B. Johnsen (Eds.), Advances in test anxiety research (Vol. 7, pp. 2-31). Lisse: Swets \& Zeitlinger.

Schwarzer, R., Mueller, J., \& Greenglass, E. (1999). Assessment of perceived general self-efficacy on the internet: Data collection in cyberspace. Anxiety, Stress and Coping: An International Journal, 12, 145-161.

Sells, I. (1980). The mathematical filter and the education of women and minorities. In L. H. Fox, L. Brody \& D. Tobin (Eds.), Women and the mathematical mystique. Baltimore, MD: Johns Hopkins University.

Silva, J. T. (2011, Julho). Fiabilidade e validade fatorial da Escala de Autoeficácia em Temas de Matemática A. Comunicação Oral apresentada no VIIII Congresso Ibero-americano de Avaliação Psicológica/XV Conferência Internacional Avaliação Psicológica: Formas e Contextos, Lisboa.

Smith, R. J., Arnkoff, D. B., \& Wright, T. L. (1990). Test anxiety and academic competence: A comparison of alternative models. Journal of Counseling Psychology, 37, 313-321.

Zeidner, M. (1998). Test anxiety: The state of the Art. New York: Plenum.

Zeidner, M. (2004). Test anxiety. In C. Spielberger (Ed.). Encyclopedia of Applied Psychology (Vol. 3, pp. 545556). Elsevier.

Zeidner, M. \& Matthews, G. (2005). Evaluation anxiety: Current theory and research. In A. J. Elliot \& C. S. Dweck (Eds.). Handbook of competence and motivation (pp. 141-163). New York: Guilford Press.

Zimmerman, B. J. (2000). Self-efficacy: An essential motive to learn. Contemporary Educational Psychology, 25, 82-91.

\section{Agradecimentos}

Os autores agradecem à Isabel Quirino, Psicóloga responsável do SPO da Escola Secundária Poeta António Aleixo (Portimão), o apoio na recolha dos dados. 